\title{
GENERAL GAUGE THEOREM FOR MULTIPLICATIVE FUNCTIONALS
}

\author{
K. L. CHUNG AND K. M. RAO
}

\begin{abstract}
We generalize our previous work on the gauge theorem and its various consequences and complements, initiated in [8] and somewhat extended by subsequent investigations (see [6]). The generalization here is two-fold. First, instead of the Brownian motion, the underlying process is now a fairly broad class of Markov processes, not necessarily having continuous paths. Second, instead of the Feynman-Kac functional, the exponential of a general class of additive functionals is treated. The case of Schrödinger operator $\Delta / 2+\nu$, where $\nu$ is a suitable measure, is a simple special case. The most general operator, not necessarily a differential one, which may arise from our potential equations is briefly discussed toward the end of the paper. Concrete instances of applications in this case should be of great interest.
\end{abstract}

1. Preliminaries. We begin with a Hunt process $\left(X_{t}, \mathcal{F}_{t}, \theta_{t} ; t \geq 0\right)$ on $(E, \mathcal{E})$ with transition semigroup $\left(P_{t}\right)$; see Chung [2] for terminology and notations.

Let

$\mathcal{E}_{+}=$the class of $\mathcal{E}$-measurable functions which are positive $(\geq 0)$.

$L^{\infty}=$ the class of bounded $\mathcal{E}$-measurable functions.

$C_{b}=$ the class of bounded continuous functions.

$C_{0}=$ the class of continuous functions vanishing at $\partial$ where $\partial$ is the one-point compactification of $E$. If open $D \subset E$, then $L^{\infty}(D)$ and $C_{b}(D)$ will denote respectively the restriction of $L^{\infty}$ and $C_{b}$ to $D$; while $C_{0}(D)$ the class of continuous functions on $D$ vanishing at $\partial_{D}=$ the one-point compactification of $D$.

For any $A \in \mathcal{E}$, let

$$
\begin{gathered}
T_{A}=\inf \left\{t>0: X_{t} \in A\right\}, \quad \tau_{A}=\inf \left\{t>0: X_{t} \in A^{C}\right\}, \\
P_{A} f(x)=E^{x}\left\{f\left(X\left(T_{A}\right)\right) ; T_{A}<\infty\right\} .
\end{gathered}
$$

We assume that $\left(P_{t}\right)$ has the strong Feller property, i.e., for each $t>0$ and $f \in L^{\infty}$, $P_{t} f \in C_{b}$. The following lemma is elementary, but will be proved.

Let $U^{\lambda}, \lambda \geq 0$, denote the $\lambda$-potential of $\left(P_{t}\right)$,

$$
U^{\lambda} f(x)=\int_{0}^{\infty} e^{-\lambda t} P_{t} f d t ; \quad 0 \leq f \in L^{\infty} .
$$

LEMMA 1. If $\left(P_{t}\right)$ has the strong Feller property, then for each $\lambda>0$, and $f \in L^{\infty}$, we have

$$
U^{\lambda} f \in C_{b}
$$

Received by the editors March 9, 1987.

1980 Mathematics Subject ('lassification (1985 Revision). Primary 60J45, 60J56; Secondary 60J40, $35 \mathrm{~J} 10$.

The first author's research was supported in part by AFOSR grant 85-0330 and NSF grant DMS 83-01072. 
Suppose there is a Radon measure $m$ on $\mathcal{E}$ such that $U^{\lambda}(x, \cdot) \ll m(\cdot)$. Let $K$ be a compact subset of $E$. Then for any $\lambda>0$ the set of measures $\left\{U^{\lambda}(x, \cdot), x \in K\right\}$ is equi-absolutely continuous with respect to $m$, namely, for any $\varepsilon>0$ there exists $\delta>0$ such that for all $A \in \mathcal{E}$ with $m(A)<\delta$ we have

$$
\sup _{x \in K} U^{\lambda}(x, A)<\varepsilon .
$$

PROOF. First, (2) is trivial by the dominated convergence theorem applied to (1). It follows from (1) and Dini's theorem that if $A_{n} \in \mathcal{E}, A_{n} \downarrow$ and $m\left(A_{n}\right) \downarrow 0$, then

$$
\sup _{x \in K} U^{\lambda}\left(x, A_{n}\right) \downarrow 0 .
$$

Now suppose (3) is false, then there exists $\varepsilon>0, x_{n} \in K, m\left(A_{n}\right)<2^{-n}$ such that $U^{\lambda}\left(x_{n}, A_{n}\right) \geq \varepsilon ;$ a fortiori,

$$
U^{\lambda}\left(x_{n}, \bigcup_{k=n}^{\infty} A_{k}\right) \geq \varepsilon .
$$

Let $x_{n} \rightarrow x \in K$, then by continuity,

$$
U^{\lambda}\left(x, \bigcup_{k=n}^{\infty} A_{k}\right) \geq \varepsilon
$$

which is a contradiction to (4).

From now on the measure $m$ in Lemma 1 will be called a reference measure. The assumption that there exists $\lambda>0$ such that for every $x, U^{\lambda}(x, \cdot)$ is absolutely continuous with respect to $m$ will hold throughout this paper. Using the resolvent equation it is seen that if this is true for some $\lambda \geq 0$, then it is true for all $\lambda \geq 0$. But we need not assume the finiteness of $U^{0}$. Lemma 1 is used to prove the next result which is essential.

LEMMA 2. Let $D$ be a relatively compact subset of $E$. For each $\varepsilon>0$ there exists $\delta>0$ such that for all $B \in \mathcal{E}, B \subset D$ with $m(B)<\delta$

$$
\sup _{x \in \bar{B}} E^{x}\left\{\tau_{B}\right\}<\varepsilon \text {. }
$$

PROOF. We have for each $x$, and $0<\varepsilon<1 / 2$,

$$
\begin{aligned}
\varepsilon \sup _{x \in \bar{D}} P^{x}\left\{\tau_{B}>\varepsilon\right\} & \leq \sup _{x \in \bar{D}} e \int_{0}^{\varepsilon} e^{-t} P^{x}\left\{\tau_{B}>t\right\} d t \\
& \leq \sup _{x \in \bar{D}} e \int_{0}^{\infty} e^{-t} P^{x}\left\{X_{t} \in B\right\} d t \\
& =e \sup _{x \in \bar{D}} U^{1}(x, B) .
\end{aligned}
$$

By Lemma 1 the last supremum is less than $\varepsilon^{2} / e$ provided that $m(B)$ is small enough. Then by the Markov property

$$
\sup _{x} P^{x}\left\{\tau_{B}>n \varepsilon\right\} \leq \varepsilon^{n}
$$


and hence for all $x$

$$
E^{x}\left\{\varepsilon^{-1} \tau_{B}\right\} \leq \sum_{0}^{\infty} P^{x}\left\{\varepsilon^{-1} \tau_{B}>n\right\} \leq \sum_{0}^{\infty} \varepsilon^{n}=(1-\varepsilon)^{-1}
$$

i.e. $E^{x}\left[\tau_{B}\right] \leq \varepsilon(1-\varepsilon)^{-1}<2 \varepsilon$.

COROLlaRY. For $t>0$, we have

$$
\sup _{x} P^{x}\left\{\tau_{B}>t\right\}<\varepsilon / t
$$

A function $f \in \mathcal{E}_{+}$is excessive iff $f \geq P_{t} f$ for $t \geq 0$ and $f=\lim _{t \downarrow 0} P_{t} f$. An excessive function is called a potential iff $P_{K^{c}} f$ converges to zero almost everywhere as the compact set $K$ increases to $E$. Let $V$ be a potential which moreover belongs to $C_{0}$. Then it is known [1, Chapter 4, Theorems 3.8 and 3.13] that $V$ has a representation as follows:

$$
V(x)=E^{x}\left\{\int_{0}^{\infty} d A_{t}\right\}=E^{x}\left\{A_{\infty}\right\}
$$

where $\left\{A_{t}, t \geq 0\right\}$ is a unique increasing continuous additive functional with $A_{0}=0$. We shall also use the notation

$$
U_{A} f(x)=E^{x}\left\{\int_{0}^{\infty} f\left(X_{t}\right) d A_{t}\right\}
$$

for suitable $f$; so that $V=U_{A} 1$.

It follows from the definition of additive functional that for $t \geq 0$ and $s \geq 0$

$$
A_{t+s}=A_{t}+A_{s} \circ \theta_{t} .
$$

Here for each $t \geq 0$ :

$$
E^{x}\left\{A_{t}\right\}=V(x)-P_{t} V(x)
$$

is continuous in $x$ because $P_{t} V$ is by the strong Feller assumption. Since $A_{t} \downarrow 0$ as $t \downarrow 0$, it follows by Dini's theorem and the assumption $V \in C_{0}$ that

$$
\lim _{t \downarrow 0} E^{x}\left\{A_{t}\right\}=0
$$

uniformly in $x \in E$.

2. Define the functional

$$
e(t)=e_{A}(t)=e^{A(t)} .
$$

Thus $\{e(t), t \geq 0\}$ is a multiplicative functional. Its first basic property is given by Lemma 3.

LEMMA 3. There exist constants $C>1$ and $b>0$ such that

$$
\sup _{x \in E} E^{x}\{e(t)\} \leq C e^{b t}
$$

ProOF. By (10), there exists $t_{0}>0$ such that

$$
\sup _{x \in E} E^{x}\left\{A_{t_{0}}\right\}<\theta<1 \text {. }
$$


Hence for all $x, 0 \leq t \leq t_{0}$, and $n \geq 2$,

$$
\begin{aligned}
\frac{1}{n} E^{x}\left\{\left(A_{t}\right)^{n}\right\} & =E^{x}\left\{\int_{0}^{t}\left(A_{t}-A_{s}\right) d\left(A_{s}\right)^{n-1}\right\} \\
& =E^{x}\left\{\int_{0}^{t} A_{t-s}\left(\theta_{s}\right) d\left(A_{s}\right)^{n-1}\right\} \\
& =E^{x}\left\{\int_{0}^{t} E^{X_{s}}\left[A_{t-s}\right] d\left(A_{s}\right)^{n-1}\right\} \\
& \leq \theta E^{x}\left\{\left(A_{t}\right)^{n-1}\right\} .
\end{aligned}
$$

Therefore, by induction we have for all $x$ :

$$
\begin{gathered}
E^{x}\left\{A_{t}^{n}\right\} \leq n ! \theta^{n}, \\
E^{x}\left\{e^{A_{t}}\right\} \leq \sum_{n=0}^{\infty} \theta^{n}=\frac{1}{1-\theta} .
\end{gathered}
$$

Since by (8),

$$
e^{A_{t+s}}=e^{A_{t}}\left(e^{A_{s}}\left(\theta_{t}\right)\right),
$$

we obtain for $(n-1) t_{0}<t \leq n t_{0}$,

$$
E^{x}\left\{e^{A_{t}}\right\} \leq 1 /(1-\theta)^{n} .
$$

This is equivalent to the assertion in (12).

COROLLARY. For any $n>0$,

$$
\lim _{t \rightarrow 0} \sup _{x \in E} E^{x}\left\{|e(t)-1|^{n}\right\}=0 .
$$

Proof of The Corollary. Observe that for $\alpha>0, e^{\alpha}-1 \leq \alpha e^{\alpha}$. Then

$$
\begin{aligned}
E^{x}\left[|e(t)-1|^{n}\right] & \leq E^{x}\left(e(t)^{n}-1\right)=E^{x}\left\{e^{n A_{t}}-1\right\} \leq E^{x}\left\{\left(n A_{t}\right) e^{n A_{t}}\right\} \\
& \leq n E^{x}\left\{A_{t}^{2}\right\}^{1 / 2}\left(E^{x}\left\{e^{2 n A_{t}}\right\}\right)^{1 / 2}
\end{aligned}
$$

Observe that $\sup _{x} E^{x}\left\{A_{t}^{2}\right\} \leq \sup _{x} 2 E^{x}\left\{A_{t}\right\}^{2} \rightarrow 0$ as $t \rightarrow 0$ by (10) and

$$
\sup _{x} \sup _{0<t<1} E^{x}\left\{e^{2 n A_{t}}\right\}<\infty
$$

by (12) applied to $2 n A$.

Lemma 2 will now be sharpened. Note first of all that if $m(B)$ is small enough, then $\tau_{B}<\infty$ a.s. We shall assume this below.

LEMMA 4. Under the conditions of Lemma 2, there exists $\delta>0$ such that if $m(B)<\delta$, then

$$
\sup _{x \in \bar{B}} E^{x}\left\{e\left(\tau_{B}\right)\right\}<\infty .
$$


Proof. If $P^{x}\left\{\tau_{B}=0\right\}=1$, then $E^{x}\left\{e\left(\tau_{B}\right)\right\}=1$. Otherwise $P^{x}\left\{\tau_{B}=0\right\}=0$ and we have

$$
\begin{aligned}
E^{x}\left\{e\left(\tau_{B}\right)\right\} & =\sum_{n=0}^{\infty} E^{x}\left\{n<\tau_{B} ; e(n) E^{X_{n}}\left\{0<\tau_{B} \leq 1 ; e\left(\tau_{B}\right)\right\}\right\} \\
& \leq \sum_{n=0}^{\infty} E^{x}\left\{n<\tau_{B} ; e(n) E^{X_{n}}\{e(1)\}\right\} \\
& \leq C_{1} \sum_{n=0}^{\infty} E^{x}\left\{n<\tau_{B} ; e(n)\right\}
\end{aligned}
$$

where

$$
C_{1}=\sup E^{x}\{e(1)\}<\infty
$$

by Lemma 3 . The sum above is bounded by

$$
\sum_{n=0}^{\infty} P^{x}\left\{n<\tau_{B}\right\}^{1 / 2} E^{x}\left\{e^{2 A(n)}\right\}^{1 / 2} .
$$

Since $\left\{2 A_{t}\right\}$ is the additive functional associated with $2 V$ which satisfies the same conditions as $V$, Lemma 3 applied to $\left\{2 A_{t}\right\}$ yields

$$
\sup _{x \in E} E^{x}\left\{e^{2 A(n)}\right\} \leq C_{2} e^{n b_{2}}
$$

with some $C_{2}>0$ and $b_{2}>0$. Hence the sum in question is bounded by

$$
\sum_{n=0}^{\infty} P^{x}\left\{n<\tau_{B}\right\}^{1 / 2} C_{2}^{1 / 2} e^{n b_{2} / 2} .
$$

Now by the Corollary of Lemma 2, there exists $\delta>0$ such that if $B \subset D$ and $m(B)<\delta$ then for all $x$

$$
P^{x}\left\{1<\tau_{B}\right\} \leq e^{-2 b_{2}} .
$$

It follows by the Markov property that

$$
P^{x}\left\{n<\tau_{B}\right\} \leq \exp \left(-2 n b_{2}\right) .
$$

Using this in (15) we see that the series converges.

The proof given above may be easily improved to show that the quantity in (14) converges to 1 as $\delta \downarrow 0$.

3. The gauge theorem. We generalize the assumptions described in $\S 1$ to a function $V=V^{(1)}-V^{(2)}$ where each $V^{(i)}, i=1,2$, satisfies the conditions for $V$ in $\S 1$. Let $A^{(i)}, i=1,2$, be the continuous increasing additive functional generating $V^{(i)}$ as in (6). Thus $A=A^{(1)}-A^{(2)}$ is a continuous additive functional generating $V$ as in (6), where $\left\{A_{t}, t \geq 0\right\}$ is of bounded variation in $[0, \infty)$ in $t$. Put

$$
A^{*}=A^{(1)}+A^{(2)} \text {. }
$$

As before we write $e(t)=e_{A}(t)=e^{A(t)}$.

Let $D$ be a nonempty open subset of $E$. We define the function

$$
g(x)=E^{x}\left\{e_{A}\left(\tau_{D}\right)\right\}, \quad x \in D .
$$


Note that we are not assuming $\tau_{D}<\infty$; on $\left\{\tau_{D}=\infty\right\},\left|A_{\infty}\right| \leq A_{\infty}^{*}$ is almost surely finite by (6). As shown above $g \in \mathcal{E}_{+}$. We call $g$ the gauge for $(D, A)$. This term was introduced in [8] in the special case when $X$ is a Brownian motion and

$$
A(t)=\int_{0}^{t} q\left(X_{s}\right) d s \quad \text { for } q \in L^{\infty}(D) .
$$

This case where $q$ is in an appropriate class of functions will be referred to later as the old case. Since then several generalizations have been given, for the latest see [6]. The gauge theorem will be proved in the present framework in two stages. In the first stage, our previous assumptions suffice but the conclusion is weaker than in previously known special cases. Another assumption will be needed to bring it to full fruition.

We begin with the process $X^{D}$ obtained by killing $X$ outside $D$. Namely, let $D \cup\{\partial\}$ be the one-point compactification of $D$, and define

$$
X_{t}^{D}= \begin{cases}X_{t} & \text { on }\left\{t<\tau_{D}\right\} \\ \partial & \text { on }\left\{t \geq \tau_{D}\right\}\end{cases}
$$

The transition semigroup of $\left\{X_{t}^{D}, t \geq 0\right\}$ will be denoted by $\left\{P_{t}^{D}\right\}$, so that

$$
P_{t}^{D} f(x)=E^{x}\left\{t<\tau_{D} ; f\left(X_{t}\right)\right\}
$$

for $f \in \mathcal{E}_{+}(D)$ with $f(\partial)=0$. We write also for $\lambda \geq 0$,

$$
U^{D, \lambda} f(x)=\int_{0}^{\infty} e^{-\lambda t} P_{t}^{D} f(x) d x=E^{x}\left\{\int_{0}^{\infty} e^{-\lambda t} f\left(X_{t}^{D}\right) d t\right\}
$$

For $\lambda>0$, this is just the Green operator for $D$. We shall call $X^{D}$ the "trace of $X$ on $D$ ". It is a Hunt process; notations such as "excessive", "finely continuous" for the trace will be prefixed by the letter $D$. We begin with an essential lemma.

LEMMA 5. The gauge function $g$ is D-finely continuous, and $g \in \mathcal{E}$.

PROOF. It is known [1, Chapter 2] that excessive functions are finely continuous, so is a difference of excessive functions. Now

$$
e\left(\tau_{D}\right)-1=\int_{0}^{\tau_{D}} \exp \left(A\left(\tau_{D}\right)-A_{t}\right) d A_{t}=\int_{0}^{\tau_{D}} \exp \left(A\left(\tau_{D}\right)\left(\theta_{t}\right)\right) d A_{t}
$$

so that if $A=A^{(1)}-A^{(2)}$,

$$
E^{\cdot}\left\{e\left(\tau_{D}\right)\right\}-1+E^{\cdot}\left\{\int_{0}^{\tau_{D}} e\left(\tau_{D}\right)\left(\theta_{t}\right) d A_{t}^{(2)}\right\}=E^{\cdot}\left\{\int_{0}^{\tau_{D}} e\left(\tau_{D}\right)\left(\theta_{t}\right) d A_{t}^{(1)}\right\},
$$

i.e.,

$$
g(x)-1+E^{\cdot}\left\{\int_{0}^{\tau_{D}} g\left(X_{t}\right) d A_{t}^{(2)}\right\}=E^{\cdot}\left\{\int_{0}^{\tau_{D}} g\left(X_{t}\right) d A_{t}^{(1)}\right\} .
$$

The last two functions are $D$-excessive. It is known that excessiveness implies nearly Borel measurability. Hence under Hypothesis (L), $g \in \mathcal{E}$ [1, Chapter 5]. Since $U^{D, \lambda} \leq U^{\lambda} \ll m$ by the assumptions of Lemma 1 , (L) holds for $X^{D}$. 
THEOREM 1. Suppose $D$ is nonempty open and relatively compact. Then the set $F=\{x \in D: g(x)<\infty\}$ is absorbing relative to $D$, namely, for any $x \in F$, $P^{x}\left\{\tau_{F}<\tau_{D}\right\}=0$. Moreover, $g$ is actually bounded on $F$.

Proof. Let $x \in F$ and $K$ be any compact subset of $D \backslash F$. By the strong Markov property

$$
\infty>g(x) \geq E^{x}\left\{T_{K}<\tau_{D} ; e^{A\left(T_{K}\right)} g\left(X\left(T_{K}\right)\right)\right\} .
$$

Since $K$ is closed, $X\left(T_{K}\right) \in K$; since $g$ is finely continuous by Lemma $5, g\left(X\left(T_{K}\right)\right)=$ $\infty$ on $\left\{T_{K}<\tau_{D}\right\}$. On the other hand, almost surely,

$$
\exp \left(A\left(T_{K}\right)\right)=\exp \left(A^{(1)}\left(T_{K}\right)-A^{(2)}\left(T_{K}\right)\right)
$$

is strictly $>0$ because $E^{x}\left\{A^{(2)}\left(T_{K}\right)\right\} \leq E^{x}\left\{A_{\infty}^{(2)}\right\}=V^{(2)}(x)<\infty$ for all $x$. It follows from (18) that $P^{x}\left\{T_{K}<\tau_{D}\right\}=0$. This being true for all compact subsets $K$ of $D \subset F$ (which belongs to $\mathcal{E}$ ), we conclude that

$$
P^{x}\left\{T_{D \backslash F}<\tau_{D}\right\}=0 .
$$

Thus $F$ is absorbing as asserted.

Next, since $m$ is a Radon measure, $m(F)<\infty$; hence we can choose $N$ so large that $B=D \cap\{N<g<\infty\}$ has measure $m(B)<\delta$, so that Lemma 4 is applicable. Then we have if $x \in B$,

$$
g(x)=E^{x}\left\{\tau_{B}=\tau_{D} ; e\left(\tau_{B}\right)\right\}+E^{x}\left\{\tau_{B}<\tau_{D} ; e\left(\tau_{B}\right) g\left(X\left(\tau_{B}\right)\right)\right\} .
$$

The first term on the right side of (19) is bounded by $M$ (say) by Lemma 4. $P^{x}$-a.s. on $\left\{\tau_{B}<\tau_{D}\right\}, X\left(\tau_{B}\right)$ does not belong to $D \backslash F$ because $F$ is absorbing, hence it must belong to the fine closure of $\{g \leq N\}$. Since $g$ is finely continuous the latter is just $\{g \leq N\}$. Therefore the second term on the right side of (19) is bounded by $M N$. It follows that on $B, g$ is bounded by $M+M N$; on $F \backslash B$ it is by $N$. The theorem is proved. (Nothing can be said of the value of $g$ on $(\partial D) \backslash\left(D^{c}\right)^{\text {reg; }}$ but see later.)

THEOREM 2. Suppose in addition to previous assumptions that for some $\lambda>0$ the set of measures

$$
\left\{U^{D, \lambda}(x, \cdot), x \in D\right\}
$$

are mutually equivalent. Then either $g \equiv \infty$ in $D$, or $g$ is bounded in $E$.

PROOF. We have proved in Theorem 1 that there exists a constant $C$ such that $F=\{g \leq C\}$. Hence $F$ is finely closed and $D \backslash F$ is finely open. If it is not empty, let $x_{0} \in D \backslash F$, then $U^{D, \lambda}\left(x_{0}, D \backslash F\right)>0$. Under the new assumption this implies $U^{D, \lambda}(x, D \backslash F)>0$ for any $x \in F$, which is impossible by Theorem 1 unless $F$ is empty. Therefore either $F$ or $D \backslash F$ is empty; if the latter, then $g$ is bounded on $D=F$ as just reiterated.

Next, let $x \in \partial D$ and not regular for $D^{c}$, then

$$
g(x)=\lim _{t \downarrow 0} E^{x}\left\{t<\tau_{D} ; e(t) g\left(X_{t}\right)\right\} \leq \lim _{t \downarrow 0} E^{x}\left\{t<\tau_{D} ; e(t)\right\} C
$$

since $g \leq C$ in $D$. By the Corollary after Lemma 3, this implies $g(x) \leq C$. For $x$ regular for $D^{c}$, of course $g(x)=1$. Hence $g$ is bounded in all of $E$. 
Let us state here the open problem of the extension of the gauge theorem to the case where $D$ is a general open set. The problem is open even in the case where

$$
A(t)=\int_{0}^{t} q\left(X_{s}\right) d s .
$$

In order to strengthen the results above we shall need the strong Feller property for $\left(P^{D}\right)$. A recent result by Chung $[3]$ states that if the original $\left(P_{t}\right)$ has both the Feller and strong Feller properties then for each nonempty open set $D,\left(P_{t}^{D}\right)$ also has both properties provided every point of $\partial D$ is regular for $D^{c}$. (We say then $D$ is regular.) In this case of course the process $X^{D}$ as well as $X$ is a Feller process, a particular case of Hunt process. As in [3] we shall call such a process "doubly Feller". Under this assumption Lemma 5 has a complement.

LEMMA 6. If $\left(P_{t}\right)$ is doubly Feller, then the gauge function $g$ is lower semicontinuous in $E$.

ProOF. Note that since $P_{t}^{D}$ is strong Feller for each bounded Borel function $f$, $E^{\cdot}\left\{t<\tau_{D} ; e(t) f\left(X_{t}\right)\right\}$ is also continuous in $D$. We have

$$
\begin{aligned}
g(x) & =\lim _{t \downarrow 0} \uparrow E^{x}\left\{t<\tau_{D} ; e\left(\tau_{D}\right)\right\}=\lim _{t \downarrow 0} E^{x}\left\{t<\tau_{D} ; e(t) g\left(X_{t}\right)\right\} \\
& =\lim _{t \downarrow 0} \uparrow \lim _{n \uparrow \infty} \uparrow E^{x}\left\{t<\tau_{D} ; e(t)\left(g\left(X_{t}\right) \wedge n\right)\right\}
\end{aligned}
$$

and the last expectations are continuous in $x$ as noted above.

Returning to Theorem 1, under the new assurnption the set $F=\{g<\infty\}=$ $\{g \leq C\}$ is closed and $D \backslash F=D \cap\{g=\infty\}$ is open. We can now give another sufficient condition for the gauge theorem.

PROPOSITION 7. Let the $D$ in Theorem 1 be also connected. Suppose for some $\lambda>0$,

$$
U^{D, \lambda}(x, d y)=u^{D, \lambda}(x, y) m(d y)
$$

and that for all $x \in D$,

$$
\varliminf_{y \rightarrow x} u^{D, \lambda}(x, y)>0 .
$$

Then either $g \equiv \infty$ in $D$ or $g$ is bounded in $D$.

ProOF. We may suppose that $F$ is not empty and prove that $D \backslash F$ is empty. Otherwise let $x_{0} \in \partial(D \backslash F) \cap D$. Then $x \in F$ since $F$ is closed. Since $F$ is absorbing with respect to $X^{D}$, we have $U^{D, \lambda}\left(x_{0}, D \backslash F\right)=0$, hence

$$
u^{D, \lambda}\left(x_{0}, y\right)=0, \quad \text { for } m \text {-a.e. } y \in D \backslash F \text {. }
$$

Let $B$ be any neighborhood of $x_{0}$; then $B \cap(D \backslash F)$ is nonempty open and so $m(B \cap(D \backslash F))>0$. Therefore (24) contradicts (23) and the proposition is proved.

The condition (23) seems contrived but actually can be easily verified in concrete cases, such as Brownian motion. More generally let us suppose that $U^{0}(x, d y)=$ $u(x, y) m(d y)$ and the following conditions are satisfied:

$$
\text { For all } x \in E: u^{0}(x, x)=\infty \text {, }
$$

For each $\varepsilon>0, u(x, y)$ is bounded for all $(x, y)$ satisfying $\rho(x, y)>$ $\varepsilon$, where $\rho$ is a metric for the topology. 
Then we have

$$
\begin{gathered}
U^{D, 0}(x, d y)=u^{D}(x, y) m(d y), \\
u^{D}(x, y)=u(x, y)-E^{x}\left\{u\left(X\left(\tau_{D}\right), y\right)\right\}
\end{gathered}
$$

and it is easy to check from (25) that

$$
\lim _{y \rightarrow x} u^{D}(x, y)=+\infty \text {. }
$$

We are indebted to Liao Ming regarding the conditions (25). Conditions similar to these in (25) were used by Chung and Rao in [8], and in Liao's dissertation (Stanford University, 1984) in a general study of potential theory.

Suppose that $\left(P_{t}\right)$ is doubly Feller. Define a semigroup $\left\{Q_{t}, t \geq 0\right\}$ as

$$
Q_{t} f(x)=E^{x}\left\{e(t) f\left(X_{t}\right)\right\}
$$

then define

$$
Q_{t}^{D} f(x)=E^{x}\left\{t<\tau_{D} ; e(t) f\left(X_{t}\right)\right\}
$$

where $D$ is nonempty, open, not necessarily relatively compact. It is proved in Chung [3] that both $\left\{Q_{t}\right\}$ and $\left\{Q_{t}^{D}\right\}$ are strongly Feller provided $\{e(t)\}$ satisfies the following conditions:

(a) for some $t>0$,

$$
\sup _{x \in E} \sup _{0 \leq s \leq t} E^{x}\{e(s)\}<\infty
$$

(b) for each $t>0$, there exists $\alpha>1$ such that

$$
\sup _{x \in E} E^{x}\left\{e(t)^{\alpha}\right\}<\infty
$$

(c) for each compact subset $K$ of $E$, we have

$$
\lim _{t \downarrow 0} \sup _{x \in K} E^{x}\{|e(t)-1|\}=0 .
$$

(d) $Q_{t}$ is doubly Feller; $Q_{t}^{D}$ is doubly Feller provided $D$ is regular.

To verify these conditions, consider $e^{*}(t)=e_{A^{*}}(t)$ where $A^{*}$ is defined in (16). Then

$$
\sup _{0 \leq s \leq t} e(s) \leq e^{*}(t)
$$

Hence (a) is true by Lemma 3 applied to $e^{*}(t)$, namely $A^{*}(t)$ instead of $A(t)$ there. Since $e(t)^{\alpha}=e_{\alpha A}(t)$, (b) is true by Lemma 3 applied to $\alpha A$ instead of $A$. (c) is proved in the Corollary after Lemma 3 , by replacing $A$ with $A^{*}$ there.

We can now sharpen Lemma 6 , indeed for a more general situation.

THEOREM 3. Suppose $\left(P_{t}\right)$ is doubly Feller, $D$ is nonempty, open and regular. If the gauge for $D$ is bounded in $D$, then it is continuous in $E$.

PROOF. Using our new notation

$$
g(x)=\lim _{t \downarrow 0} Q_{t}^{D} g(x)
$$

where for each $t>0, Q_{t}^{D} g \in C_{b}$ because $\left\{Q_{t}^{D}\right\}$ has the strong Feller property and $g$ is assumed to be bounded. Now

$$
\begin{aligned}
0 & \leq g(x)-Q_{t}^{D} g(x)=P^{x}\left\{t \geq \tau_{D} ; e\left(\tau_{D}\right)\right\} \leq P^{x}\left\{t \geq \tau_{D} ; e^{*}(t)\right\} \\
& \leq P^{x}\left\{t \geq \tau_{D}\right\}^{1 / 2} E^{x}\left\{e^{2 A^{*}(t)}\right\}^{1 / 2}
\end{aligned}
$$


By Lemma 3 applied to $2 A^{*}$,

$$
\sup _{x \in E} E^{x}\left\{e^{2 A^{*}(t)}\right\} \leq C(t)<\infty
$$

where $C(t)$ is a constant depending on $t$. Since $\left(P_{t}\right)$ is doubly Feller,

$$
\lim _{t \downarrow 0} \sup _{x \in K} P^{x}\left\{t \geq \tau_{D}\right\}=0
$$

for each compact $K$ in $D$; see Lemma 2 of [3], the crucial Lemma there. It follows from (26) that $Q_{t}^{D} g$ converges uniformly to $g$ in each $K$, and so $g$ is continuous in $D$.

Since $D$ is regular, $g=1$ on $D^{c}$. It remains to prove that as $x \in D, x \rightarrow z \in \partial D$, $g(x)$ converges to 1 . Indeed

$$
\begin{aligned}
g(x)-1 & =E^{x}\left\{e\left(\tau_{D}\right)-1 ; \tau_{D} \leq t\right\}+E^{x}\left\{e\left(\tau_{D}\right)-1 ; t<\tau_{D}\right\} \\
|g(x)-1| & \leq E^{x}\left\{e_{A^{*}}(t)-1\right\}+E^{x}\left\{\left|e(t) g\left(X_{t}\right)-1\right| ; t<\tau_{D}\right\} .
\end{aligned}
$$

The first term tends to zero as $t \rightarrow 0$ by Lemma 3 applied to $A^{*}$. For the second term we have

$$
E^{x}\left\{\left|e(t) g\left(X_{t}\right)-1\right| ; t<\tau_{D}\right\} \leq C E^{x}\left\{e^{2}(t)\right\}^{1 / 2}\left(P^{x}\left(t<\tau_{D}\right)\right)^{1 / 2}+P^{x}\left\{t<\tau_{D}\right\}
$$

since $g \leq C$, $\sup _{x} \sup _{0 \leq t \leq 1} E^{x}\left\{e(t)^{2}\right\}<\infty$, and $P^{x}\left(t<\tau_{D}\right) \rightarrow 0$ as $x \rightarrow \partial D$, the second term also tends to zero.

The following complement to the gauge theorem is easy but important. It does not depend on the latter and holds for any open set $D$.

PROPOSITION 8. $\inf _{x \in E} g(x)>0$.

ProOF. By Jensen's inequality

$$
g(x) \geq E^{x}\left\{e^{-A^{(2)}\left(\tau_{D}\right)}\right\} \geq e^{-E^{x}\left\{A^{(2)}\left(\tau_{D}\right)\right\}} .
$$

Since

$$
\sup _{x \in E} E^{x}\left\{A^{(2)}\left(\tau_{D}\right)\right\} \leq \sup _{x \in E} V^{(2)}(x)<\infty,
$$

the proposition follows.

4. Consequences of bounded gauge. In this section we assume that the gauge for $(D, A)$ is bounded, when $D$ is a nonempty open set, not necessarily relatively compact. We shall deduce several consequences from this, some of which are strictly stronger than the initial assumptions. Note that the boundedness of the gauge for $(D, A)$ does not imply that for $\left(D, A^{*}\right)$. Nevertheless we have the following useful result.

THEOREM 4. We have

$$
\sup _{x \in E} E^{x}\left\{\int_{0}^{\tau_{D}} e_{A}(t) d A_{t}^{*}\right\}<\infty .
$$

PROOF. We begin with the calculus formula

$$
\int_{0}^{s} e^{A^{*}(t)} d A^{*}(t)=e^{A^{*}(s)}-1
$$


Since $A(t) \leq A^{*}(t)$ it follows that

$$
E^{x}\left\{\int_{0}^{t} e_{A}(t) d A^{*}(t)\right\} \leq E^{x}\left\{e_{A^{*}}(t)\right\}-1 \leq C(t)
$$

where $C(t)$ is a constant depending on $t$ but not $x$, by Lemma 3 applied to $A^{*}$. Now we have, writing $\tau$ for $\tau_{D}$ and $e(t)$ for $e_{A}(t)$ below,

$$
\begin{aligned}
E^{x}\left\{\int_{0}^{\tau} e(t) d A_{t}^{*}\right\} & =E^{x}\left\{\sum_{n=0}^{\infty} \int_{\tau \wedge n}^{\tau \wedge(n+1)} e(t) d A^{*}(t)\right\} \\
& =\sum_{n=0}^{\infty} E^{x}\left\{n<\tau ; \int_{n}^{\tau \wedge(n+1)} e(t) d A_{t}^{*}\right\} \\
& =\sum_{n=0}^{\infty} E^{x}\left\{n<\tau ; e(n) E^{X_{n}}\left\{\int_{0}^{\tau \wedge 1} e(t) d A_{t}^{*}\right\}\right\} \\
& \leq C(1) \sum_{n=0}^{\infty} E^{x}\{n<\tau ; e(n)\}
\end{aligned}
$$

by (28). The convergence of the series in (29) is proved in the following lemma.

LEMMA 9. For every $\delta>0$, there exists a constant $C(\delta)$ such that

$$
\|g\|_{\infty}^{-1} g(x) \leq \sum_{n=0}^{\infty} E^{x}\{n \delta<\tau ; e(n \delta)\} \leq C(\delta) .
$$

PROOF. Since the proof is the same for any $\delta>0$ we take $\delta=1$. For each $x \in D$ the quantity $E^{x}\{n<\tau ; e(\tau)\}$ decreases to zero. Hence for any $\varepsilon>0$, we can choose $N$ and a set $F$ such that $\sup _{x \in F} E^{x}\{N<\tau ; e(\tau)\}<\varepsilon$ and $m(D \backslash F)<\varepsilon$. By choosing a compact subset of $F$ if necessary we may assume that $F$ itself is compact. Here $\varepsilon$ is such that $\sup _{x} E^{x}\left\{\left(e\left(\tau_{1}\right)\right)^{2}\right\} \leq 2$ where $\tau_{1}=\tau_{D \backslash F}$. This is possible by the remark immediately after the proof of Lemma 4 . We have

$$
\begin{aligned}
& E^{x}\{2 N<\tau ; e(\tau)\} \leq E^{x}\left\{N<\tau_{1} ; e(\tau)\right\}+E^{x}\left\{\tau_{1} \leq N, 2 N<\tau ; e(\tau)\right\} \\
& \quad \leq\|g\|_{\infty} E^{x}\left\{N<\tau_{1} ; e\left(\tau_{1}\right)\right\}+E^{x}\left\{\tau_{1}<\tau ; e\left(\tau_{1}\right) E^{X\left(\tau_{1}\right)}[N<\tau ; e(\tau)]\right\} .
\end{aligned}
$$

The first term above can be estimated by $E^{x}\left\{e^{2}\left(\tau_{1}\right)\right\}^{1 / 2} P^{x}\left(\tau_{1}>N\right)^{1 / 2}$ which is uniformly small if $N$ is large, by Corollary to Lemma 2. On the set $\left\{\tau_{1}<\tau\right\}$, $X_{\tau_{1}} \in F$ so that the second is bounded by $2 \varepsilon$. Thus for large $N$,

$$
\sup _{x} E^{x}\{N<\tau ; e(\tau)\}
$$

is small. Now from Proposition $8, g$ is bounded from below, say $g \geq m$. Then

$$
\begin{aligned}
E^{x}\{N<\tau ; e(N)\} & \leq \frac{1}{m} E^{x}\left\{N<\tau ; e(N) g\left(X_{N}\right)\right\} \\
& \leq \frac{1}{m} E^{x}\{N<\tau ; e(\tau)\} .
\end{aligned}
$$

Therefore we see that for $N$ large enough

$$
\sup _{x} E^{x}\{N<\tau ; e(N)\} \leq \alpha<1
$$


By the Markov property for any $k$ :

$$
\sup _{x} E^{x}\{k N<\tau ; e(k N)\} \leq \alpha^{k} .
$$

Further if $j<N$ :

$$
\begin{aligned}
E^{x}\{k N+j<\tau ; e(k N+j)\} & =E^{x}\left\{j<\tau ; e(j) E^{X_{j}}\{k N<\tau ; e(k N)\}\right\} \\
& \leq \alpha^{k} \sup _{x, j \leq N} E^{x}\{e(j)\}=\alpha^{k} \mathcal{M} .
\end{aligned}
$$

All these inequalities give

$$
\begin{aligned}
\sum_{n} E^{x}\{n<\tau ; e(n)\} & =\sum_{k=0}^{\infty} \sum_{j=1}^{N} E^{x}\{k N+j<\tau ; e(k N+j)\} \\
& \leq N \mathcal{M} \sum_{0}^{\infty} \alpha^{k}=\frac{N \mathcal{M}}{1-\alpha}=C(1) .
\end{aligned}
$$

Finally we have

$$
\begin{aligned}
g(x) & =\sum E^{x}[n \delta<\tau<(n+1) \delta ; e(\tau)] \\
& \leq \sum E^{x}\left[n \delta<\tau ; e(n \delta) g\left(X_{n \delta}\right)\right] \leq\|g\|_{\infty} \sum E^{x}[n \delta<\tau ; e(n \delta)]
\end{aligned}
$$

which proves the result.

The following consequence is stronger than the gauge theorem.

THEOREM 5 .

$$
\sup _{x \in D} E^{x}\left\{\sup _{0 \leq t \leq \tau_{D}} e_{A}(t)\right\}<\infty
$$

ProOF. We have

$$
e^{A(t)}=1+\int_{0}^{t} e_{A}(s) d A(s) \leq 1+\int_{0}^{t} e_{A}(s) d A^{*}(s)
$$

hence

$$
\sup _{0 \leq t \leq \tau_{D}} e_{A}(t) \leq 1+\int_{0}^{\tau_{D}} e_{A}(s) d A^{*}(s)
$$

and (31) follows from (27).

COROLlary.

$$
g(x)=E^{x}\left[\int_{0}^{\tau_{D}} e(t) d A_{t}\right]+1 .
$$

PROOF. We can use Fubini by (27).

Before we proceed let us state several properties that are equivalent to the boundedness of the gauge. These are similar to Theorem 3.2 in [8] but somewhat improved. Recall the notation $Q_{t}^{D}$ from $\S 3$.

THEOREM 6. Any one of the following propositions is equivalent to the boundedness of the gauge.

(i) For some $\delta>0$ and some $x$,

$$
\sum_{n} Q_{n \delta}^{D} 1(x)<\infty .
$$


(ii) For all $\delta>0$, and all $x$, (32) is true.

(iii) For some $x$,

$$
\int_{0}^{\infty} Q_{t}^{D} 1(x) d t<\infty
$$

(iv) There exists $t$ such that

$$
\sup _{x \in D} Q_{t}^{D} 1(x)<1 .
$$

(v) There exists $C>0$ and $b>0$ such that

$$
\sup _{x \in D} Q_{t}^{D} 1(x) \leq C e^{-b t} .
$$

Proof. That (iv) and (v) are equivalent is by the usual use of the Markov property and (v) clearly implies (i). Of course (ii) implies (i). In short, the following equivalences are clear or easy:

$$
\text { (iv) } \Leftrightarrow(\mathrm{v}) ; \text { (v) } \Rightarrow \text { (i); (ii) } \Rightarrow \text { (i). }
$$

If (i) is valid then $g(x)<\infty$ and hence bounded by the Gauge Theorem. By Lemma 9 then (ii) is valid and in the course of the proof of Lemma 9 we also saw that (iv) is valid. We now show that (i) is equivalent to (iii).

Fix $x$ and suppose (32) is true. We have

$$
\begin{aligned}
\int_{0}^{\infty} Q_{t} 1(x) d t & =\sum_{0}^{\infty} \int_{n \delta}^{n \delta+\delta} Q_{t} 1(x) d t=\sum_{0}^{\infty} \int_{n \delta}^{n \delta+\delta} E^{x}[t<\tau ; e(t)] d t \\
& =\sum_{0}^{\infty} \int_{n \delta}^{n \delta+\delta} E^{x}\left[n \delta<\tau ; e(n \delta) E^{X(n \delta)}[t-n \delta<\tau ; e(t-n \delta)]\right] d t \\
& \leq C \sum_{0}^{\infty} E^{x}[n \delta<\tau ; e(n \delta)]<\infty
\end{aligned}
$$

where $C=\sup _{x} E^{x}\left\{e_{A^{*}}(1)\right\}$.

Suppose now that (iii) holds. For $n \delta<t<(n+1) \delta$ we have

$$
\begin{aligned}
Q_{(n+1) \delta} 1(x) & =E^{x}[(n+1) \delta<\tau ; e(n \delta+\delta)] \leq E^{x}[t<\tau ; e(n \delta+\delta)] \\
& =E^{x}\left[t<\tau ; e(t) E^{X_{t}}[e((n+1) \delta-t)]\right] \leq C E^{x}[t<\tau ; e(t)]
\end{aligned}
$$

where $C$ is as before. Hence

$$
Q_{n \delta+\delta} 1(x) \leq C \delta^{-1} \int_{n \delta}^{n \delta+\delta} Q_{t} 1(x) d t
$$

and the quantity in (i) is bounded by $1+C \delta^{-1} \int_{0}^{\infty} Q_{t} 1(x) d t$. Thus all five are equivalent to the boundedness of the gauge.

5. The super gauge theorem. This is another consequence of the boundedness of the gauge, which asserts that if the gauge for $(D, A)$ is bounded then so is it for $(D,(1+\varepsilon) A)$ for sufficiently small $\varepsilon>0$. This phenomenon has an intuitive base in the old case, having to do with eigenvalues (spectrum) of an associated Schrödinger operator. See $\S 6$. We shall give two distinct proofs of this interesting result. The first is more direct and similar to the old treatment. The second relates it to the recent development in "bounded mean oscillations (BMO)" and is included here on account of this amusing connection. 
THEOREM 7. If the gauge is bounded (in $E$ ) then there exists $\varepsilon>0$ such that

$$
\sup _{x \in E} E^{x}\left\{e\left(\tau_{D}\right)^{1+\varepsilon}\right\}<\infty .
$$

ProOF. By Theorem 6, there exists $t$ such that for all $x \in D$,

$$
Q_{t}^{D} 1(x)=E^{x}\left\{t<\tau_{D} ; e(t)\right\}<1 .
$$

Consider

$$
\begin{aligned}
E^{x}\left\{\left|e(t)^{1+\varepsilon}-e(t)\right|\right\} & =E^{x}\left\{e(t)\left|e(t)^{\varepsilon}-1\right|\right\} \\
& \leq E^{x}\left\{e(t)^{2}\right\}^{1 / 2} E^{x}\left\{\left|e^{\varepsilon A(t)}-1\right|^{2}\right\}^{1 / 2} \\
& \leq C(t) E^{x}\left\{\left|e^{\varepsilon A(t)}-1\right|^{2}\right\}^{1 / 2},
\end{aligned}
$$

where $C(t)$ is a constant independent of $x$, by Lemma 3 applied to $2 A$. We have

$$
\left|e^{\varepsilon A(t)}-1\right| \leq e^{\varepsilon A^{*}(t)}-1 .
$$

Now since $A^{*}$ is increasing

$$
e^{\varepsilon A^{*}(t)}-1=\int_{0}^{t} e^{\varepsilon A^{*}(s)} \varepsilon d A^{*}(s) \leq \varepsilon e^{\varepsilon A^{*}(t)} A^{*}(t) .
$$

Hence

$$
E^{x}\left\{\left(e^{\varepsilon A^{*}(t)}-1\right)^{2}\right\} \leq \varepsilon^{2} E^{x}\left\{e^{2 \varepsilon A^{*}(t)} A^{*}(t)^{2}\right\} \leq \varepsilon^{2} C(t)
$$

by Lemma 3. It follows from this and (35) that as $\varepsilon \downarrow 0, E^{x}\left\{\left|e(t)^{1+\varepsilon}-e(t)\right|\right\}$ converges to zero uniformly in $x \in E$. Therefore by (34), if $\varepsilon$ is sufficiently small

$$
\sup _{x \in E} E^{x}\left\{t<\tau_{D} ; e(t)^{1+\varepsilon}\right\}<1 .
$$

But $e_{A}(t)^{1+\varepsilon}=e_{(1+\varepsilon) A}(t)$. Hence by Theorem 6(iv) applied to $(1+\varepsilon) A$ we conclude that the gauge for $(D,(1+\varepsilon) A)$ is bounded.

For the second proof of Theorem 7 we introduce the probability measure, for $\Lambda \in \mathcal{F}_{\infty}$,

$$
M^{x}(\Lambda)=\frac{1}{g(x)} E^{x}\left\{e_{A}\left(\tau_{D}\right) 1_{\Lambda}\right\}, \quad x \in E,
$$

where $g$ is the gauge for $(D, A)$. This is well defined since $g>0$ by Proposition 8 , and $M^{x}(Q)=1$ since $g(x)<\infty$. Now we compute the conditional expectation. Put $\mathcal{F}_{t}^{\tau}=\mathcal{F}_{t} 1_{(t<\tau)}$ where $\tau=\tau_{D}$,

$$
\begin{aligned}
M^{x}\left\{A_{\tau}^{*}-A_{t}^{*} \mid \mathcal{F}_{t}\right\} & =M^{x}\left\{A_{\tau}^{*}\left(\theta_{t}\right) \mid \mathcal{F}_{t}^{\tau}\right\} \\
& =\frac{1}{g(x)} E^{x}\left\{e(t)\left(e(\tau) A_{\tau}^{*}\right)\left(\theta_{t}\right) \mid \mathcal{F}_{t}^{\tau}\right\} \\
& =\frac{1}{g(x)} E^{x}\left\{t<\tau ; e(t) E^{X_{t}}\left\{e(\tau) A_{\tau}^{*}\right\} \mid \mathcal{F}_{t}^{\tau}\right\}
\end{aligned}
$$

But for all $x \in D$,

$$
\begin{aligned}
E^{x}\left\{e(\tau) A_{\tau}^{*}\right\} & =E^{x}\left\{\int_{0}^{\tau} e(\tau) d A_{t}^{*}\right\}=E^{x}\left\{\int_{0}^{\tau} e(t) E^{X_{t}}\{e(\tau)\} d A_{t}^{*}\right\} \\
& \leq\|g\|_{\infty} E^{x}\left\{\int_{0}^{\tau} e(t) d A_{t}^{*}\right\} \leq C_{1}
\end{aligned}
$$


by Theorem 4 . Hence the quantity in (37) is bounded by

$$
\frac{1}{g(x)} E^{x}\{t<\tau ; e(t)\} C_{1} \leq \frac{1}{C_{2}} E^{x}\left\{\sup _{0 \leq t \leq \tau} e(t)\right\} C_{1}
$$

where $C_{2}$ is a lower bound for $g$ by Proposition 8 . By Theorem 5 , the last quantity is bounded in $D$. Thus there exists a constant $C$ such that for all $x \in D$,

$$
M^{x}\left\{A_{\tau}^{*}-A_{t}^{*} \mid \mathcal{F}_{t}^{\tau}\right\}<C .
$$

Now replace $A^{*}$ by $\varepsilon A^{*}$ above, then for $\varepsilon<C^{-1}$, we obtain

$$
M^{x}\left\{\varepsilon\left(A_{\tau}^{*}-A_{t}^{*}\right) \mid \mathcal{F}_{t}^{\tau}\right\}<\theta<1 .
$$

It follows by Theorem 109 (John-Nirenberg) in [9, p. 193] that

$$
E^{x}\left\{e_{(1+\varepsilon) A}(\tau)\right\}=E^{x}\left\{e(\tau) e_{\varepsilon A^{*}}(\tau)\right\}=g(x) M^{x}\left\{e^{\varepsilon A_{\tau}^{*}}\right\}<\infty .
$$

A curious consequence of the super gauge theorem is that either there exists $\varepsilon_{0}>0$ such that the gauge for $(D,(1+\varepsilon) A)$ is bounded for all $\varepsilon<\varepsilon_{0}$, but infinite for $\varepsilon=\varepsilon_{0}$, or it is bounded for $(D, p A)$ for all $p>0$. The latter holds e.g. if $A=-A^{(2)}$. Let us add that by the super gauge theorem, all previous results such as Theorems 4 and 5 are valid when the $A$ there is replaced by $(1+\varepsilon) A$ for sufficiently small $\varepsilon>0$. For instance,

$$
\sup _{x \in D} E^{x}\left\{\sup _{0 \leq t \leq \tau_{0}} e_{A}(t)^{1+\varepsilon}\right\}<\infty .
$$

An interesting application of the super gauge theorem will be given in $\S 6$. For another application in the old case see Chung [4].

6. Green potentials. We call the function

$$
U^{D} f(x)=E^{x}\left\{\int_{0}^{\tau_{D}} f\left(X_{t}\right) d t\right\}=\int_{0}^{\infty} P_{t}^{D} f(x) d t, \quad f \in L^{\infty}(D),
$$

the Green potential of $f$. Indeed if $X$ is the Brownian motion in $R^{d}, d \geq 1$, this is the classical Green potential. Here we need $D$ to be relatively compact to ensure that $\tau_{D}<\infty$ a.s.; otherwise we have to consider $U^{D, \lambda}$ for $\lambda>0$ as in $\S 1$. For the sake of simplicity we shall assume (for the first time in this paper) that $X$ is transient in the sense that for each compact subset $K$ of $E$,

$$
x \rightarrow U(x, K)=E^{x}\left\{\int_{0}^{\infty} 1_{K}\left(X_{t}\right) d t\right\}
$$

is a bounded function in $E$. Then it follows that for all relatively compact open sets $D \sup _{x \in E} E^{x}\left\{\tau_{D}\right\}<\infty$, so that the Green potential in (38) is bounded in $E$. Now, with the additive functional $A$ specified above, we generalize the notion as follows:

$$
V^{D} f(x)=E^{x}\left\{\int_{0}^{\tau_{D}} e_{A}(t) f\left(X_{t}\right) d t\right\}=\int_{0}^{\infty} Q_{t}^{D} f(x) d t .
$$

[Note that this expression is given in Theorem 6, (iii).] This will be called the $A$-Green function, so that $U^{D}$ becomes the $O$-Green potential. There is a simple relation between these, given in the old case in Chung [5]. It is easily extended to the present more general case; but we shall spell out half of it below. 
THEOREM 8. Assume the gauge is bounded. We have

$$
\begin{aligned}
V^{D} f & =U^{D} f+E \cdot\left\{\int_{0}^{\tau_{D}} e_{A}(s) U^{D} f\left(X_{s}\right) d A_{s}\right\} \\
& =U^{D} f+E^{\cdot}\left\{\int_{0}^{\tau_{D}} V^{D} f\left(X_{s}\right) d A_{s}\right\} .
\end{aligned}
$$

PROOF. We may assume $f \in L_{+}^{\infty}(D)$. As just remarked, $U^{D} f$ is bounded. We have, writing $\tau$ for $\tau_{D}, e(t)$ for $e_{A}(t)$ :

$$
\begin{aligned}
E^{x}\left\{\int_{0}^{\tau} e(s) U^{D} f\left(X_{s}\right) d A_{s}\right\} & =E^{x}\left\{\int_{0}^{\tau} e(s) E^{X_{s}}\left\{\int_{0}^{\tau} f\left(X_{r}\right) d r\right\} d A_{s}\right\} \\
& =E^{x}\left\{\int_{0}^{\tau} e(s) \int_{s}^{\tau} f\left(X_{r}\right) d r d A_{s}\right\} .
\end{aligned}
$$

In order to reverse the order of integration above, we note that $\left|d A_{s}\right| \leq d A_{s}^{*}$, and

$$
E^{x}\left\{\int_{0}^{\tau} e(s) \int_{s}^{\tau} f\left(X_{r}\right) d r d A_{s}^{*}\right\}=E^{x}\left\{\int_{0}^{\tau} e(s) U^{D} f\left(X_{s}\right) d A_{s}^{*}\right\}<\infty
$$

by Theorem 4. Therefore, we can reverse the order of integration in (42) and obtain

$$
\begin{aligned}
E^{x}\left\{\int_{0}^{\tau} f\left(X_{r}\right) \int_{0}^{r} e(s) d A_{s} d r\right\} & =E^{x}\left\{\int_{0}^{\tau} f\left(X_{r}\right)[e(r)-1] d r\right\} \\
& =V^{D} f(x)-U^{D} f(x) .
\end{aligned}
$$

This establishes the first relation in (41); the second is similar.

Theorem 8 is the integrated form of relations between the infinitesimal generators of the semigroups $\left(P_{t}^{D}\right)$ and $\left(Q_{t}^{D}\right)$. In the old case, when $X$ is the Brownian motion, and

$$
A(t)=\int_{0}^{t} q\left(X_{s}\right) d s
$$

for an appropriate class of functions $q$, these are the Laplacian $\Delta / 2$ and Schrödinger operators $\Delta / 2+q$, respectively, and $(\Delta / 2+q) V^{D} f=-f$.

In the general case recalling (6) and $(7)$ of $\S 1$, we define the Revuz measure (see [10]) associated with $U_{A} 1$ as follows: for $\phi \in L^{\infty}$

$$
\mu_{A}(\phi)=\lim _{t \downarrow 0} t^{-1} \int_{\mathcal{E}} E^{x}\left\{\int_{0}^{t} \phi\left(X_{s}\right) d A_{s}\right\} m(d x)
$$

where $m$ is a reference measure. Then the Revuz measure associated with the $U_{A} f$ in (7) is given by $f \cdot \mu_{A}$. In the old case where $A(t)=\int_{0}^{t} q\left(X_{s}\right) d s$ we have $\mu_{A}=q \cdot m$. Now put $u=V^{D} f$, and

$$
B_{t}=\int_{0}^{t} f\left(X_{s}^{D}\right) d s+\int_{0}^{t} u\left(X_{s}^{D}\right) d A_{s}
$$

Then by the second relation in (41), we have

$$
u(x)=E^{x}\left\{B_{\infty}\right\}, \quad x \in D .
$$

Hence the Revuz measure associated with $u$ is given by

$$
\mu_{B}=f \cdot m+u \cdot \mu_{A} .
$$


In the old case this reduces to

$$
\mu_{B}=(f+u q) \cdot m \text {. }
$$

Since in that case $q$ is also given by

$$
q=\left(-2^{-1} \Delta\right) U_{A} 1
$$

this shows

$$
-2^{-1} \Delta u=f+u q
$$

or

$$
\left(2^{-1} \Delta+q\right) u=-f
$$

namely that $u$ is a solution of the nonhomogeneous Schrödinger equation above. In the cases where the Laplacian operator in (44) is replaced by a more general differential operator, an equation similar to (45) results. Moreover, the old case may be slightly generalized so that the measure $q \cdot m$ becomes a Radon measure $\nu$. That is an easy consequence of our general theorem applied to the additive functional generated by $U \nu$.

A remarkable connection between the gauge and $A$-Green potential will now be discussed. Consider the functions for $f \in L_{+}^{\infty}(D)$ and $x \in D$,

$$
\begin{aligned}
\phi(x) & =E^{x}\left\{e\left(\tau_{D}\right) \int_{0}^{\tau_{D}} f\left(X_{t}\right) d t\right\} \\
& =E^{x}\left\{\int_{0}^{\tau_{D}} f\left(X_{t}\right) E^{X_{t}}\left[e\left(\tau_{D}\right)\right] d t\right\}=E^{x}\left\{\int_{0}^{\tau_{D}} e(t) g\left(X_{t}\right) f\left(X_{t}\right) d t\right\} .
\end{aligned}
$$

It follows at once by Proposition 8 that when the gauge is bounded $\phi(x) \sim V^{D} f(x)$ where for two positive functions $\phi_{1}$ and $\phi_{2}$ we use the notation $\phi_{1} \sim \phi_{2}$ to denote that $\phi_{1} / \phi_{2}$ is both bounded above and away from zero.

Next by the super gauge theorem, for sufficiently small $\varepsilon$ we have

$$
\sup _{x} E^{x}\left\{e\left(\tau_{D}\right)^{1+\varepsilon}\right\} \leq M_{1}<\infty .
$$

Now by Hölder's inequality,

$$
\phi(x) \leq E^{x}\left\{e\left(\tau_{D}\right)^{1+\varepsilon}\right\}^{1 /(1+\varepsilon)} E^{x}\left\{\left(\int_{0}^{\tau_{D}} f\left(X_{t}\right) d t\right)^{(1+\varepsilon) / \varepsilon}\right\}^{\varepsilon /(1+\varepsilon)} .
$$

We may choose $\varepsilon$ so that $(1+\varepsilon) / \varepsilon=p$ is an even integer. Then by an argument we have used before in the proof of Lemma 3, if

$$
\sup _{x \in E} U^{D} f(x)=M_{2}<\infty
$$

we have

$$
E^{x}\left\{\left(\int_{0}^{\tau_{D}} f\left(X_{t}\right) d t\right)^{p}\right\} \leq p ! M_{2}^{p}
$$

Consequently there exists $M$ :

$$
\sup _{x \in E} \phi(x) \leq M \sup _{x \in E} U^{D} f(x),
$$

where $M=M_{1}(p !)^{1 / p}$. $\left(M_{1}\right.$ depends on $p$. $)$ 
In the old case when $X$ is the Brownian motion and

$$
A(t)=\int_{0}^{t} q\left(X_{s}\right) d s
$$

and when $\partial D$ is smooth, say of the class $C^{1,1}$, Zhao [6] proved that there exists $M$ such that for all $x, \phi(x) \leq M U^{D} f(x)$. The result above is a weaker version of this but valid in our general case without any assumption on $\partial D$.

\section{REFERENCES}

1. R. M. Blumenthal and R. K. Getoor, Markov processes and potential theory, Academic Press, New York, 1968.

2. K. L. Chung, Lectures from Markov processes to Brownian motion, Springer-Verlag, Berlin and New York, 1985.

3. _ Doubly Feller process with multiplicative functional, Seminar on Stochastic Processes, Birkhauser, 1985, pp. 63-78.

4. __ Properties of finite gauge with an application to local time, Essays in honor of Carl-Gustav Esseen, Uppsala, Sweden, 1983, pp. 16-24.

5. _ _ Notes on the inhomogeneous Schrödinger equation, Seminar on Stochastic Processes, Birkhäuser, 1984, pp. 55-62.

6. K. L. Chung and Z. Zhao, forthcoming monograph.

7. K. L. Chung and M. Rao, A new setting for potential theory, Ann. Inst. Fourier (Grenoble) 30 (1980), 167-198.

8. __ Feynman-Kac functional and the Schrödinger equation, Seminar on Stochastic Processes, Birkhäuser, 1981, pp. 1-29.

9. C. Dellacheri and P. A. Meyer, Probabilités et potentiel, Hermann, Paris, 1980.

10. D. Revuz, Mesures associees aux fonctionelles additives de Markov. I, Trans. Amer. Math. Soc. 148 (1970), 501-531. 94305

Department of Mathematics, Stanford University, Stanford, California

Department of Mathematics, University of Florida, Gainesville, Florida 32611 